\title{
Safety assessment of near infrared light emitting diodes for diffuse optical measurements Alper Bozkurt* and Banu Onaral
}

\author{
Address: School of Biomedical Engineering, Science and Health Systems, Drexel University, 3141 Chestnut Street, Philadelphia, Pennsylvania \\ 19104, USA \\ Email: Alper Bozkurt* - alper.bozkurt@drexel.edu; Banu Onaral - banu.onaral@drexel.edu \\ * Corresponding author
}

Published: 22 March 2004

BioMedical Engineering OnLine 2004, 3:9
Received: 30 January 2004

Accepted: 22 March 2004

This article is available from: http://www.biomedical-engineering-online.com/content/3/1/9

(C) 2004 Bozkurt and Onaral; licensee BioMed Central Ltd. This is an Open Access article: verbatim copying and redistribution of this article are permitted in all media for any purpose, provided this notice is preserved along with the article's original URL.

\begin{abstract}
Background: Near infrared (NIR) light has been used widely to monitor important hemodynamic parameters in tissue non-invasively. Pulse oximetry, near infrared spectroscopy, and diffuse optical tomography are examples of such NIR light-based applications. These and other similar applications employ either lasers or light emitting diodes (LED) as the source of the NIR light. Although the hazards of laser sources have been addressed in regulations, the risk of LED sources in such applications is still unknown.
\end{abstract}

Methods: Temperature increase of the human skin caused by near infrared LED has been measured by means of in-vivo and in-vitro experiments. Effects of the conducted and radiated heat in the temperature increase have been analyzed separately.

Results: Elevations in skin temperature up to $10^{\circ} \mathrm{C}$ have been observed. The effect of radiated heat due to NIR absorption is low - less than $0.5^{\circ} \mathrm{C}$ - since emitted light power is comparable to the NIR part of sunlight. The conducted heat due to semiconductor junction of the LED can cause temperature increases up to $9^{\circ} \mathrm{C}$. It has been shown that adjusting operational parameters by amplitude modulating or time multiplexing the LED decreases the temperature increase of the skin significantly.

Conclusion: In this study, we demonstrate that the major risk source of the LED in direct contact with skin is the conducted heat of the LED semiconductor junction, which may cause serious skin burns. Adjusting operational parameters by amplitude modulating or time multiplexing the LED can keep the LED within safe temperature ranges.

\section{Background}

New medical applications using optical measurement techniques are emerging rapidly. These methods are used to study the content of biological pigments and tissue structures by analyzing the absorption and scattering of the induced light. When visible or near infrared light at specific wavelengths in the window of 600 to $950 \mathrm{~nm}$ (fig. 1 ) is shone through the tissue, information about the amount of blood chromophores such as oxyhemoglobin $(\mathrm{HbO} 2)$ and deoxyhemoglobin $(\mathrm{Hb})$ can be obtained. This constitutes an important measure of the hemodynamic state of the tissue [1], and is the principle upon which pulse oximetry is based.

The hazard potential of the near-infrared light should be considered from two perspectives: eye hazards and skin 


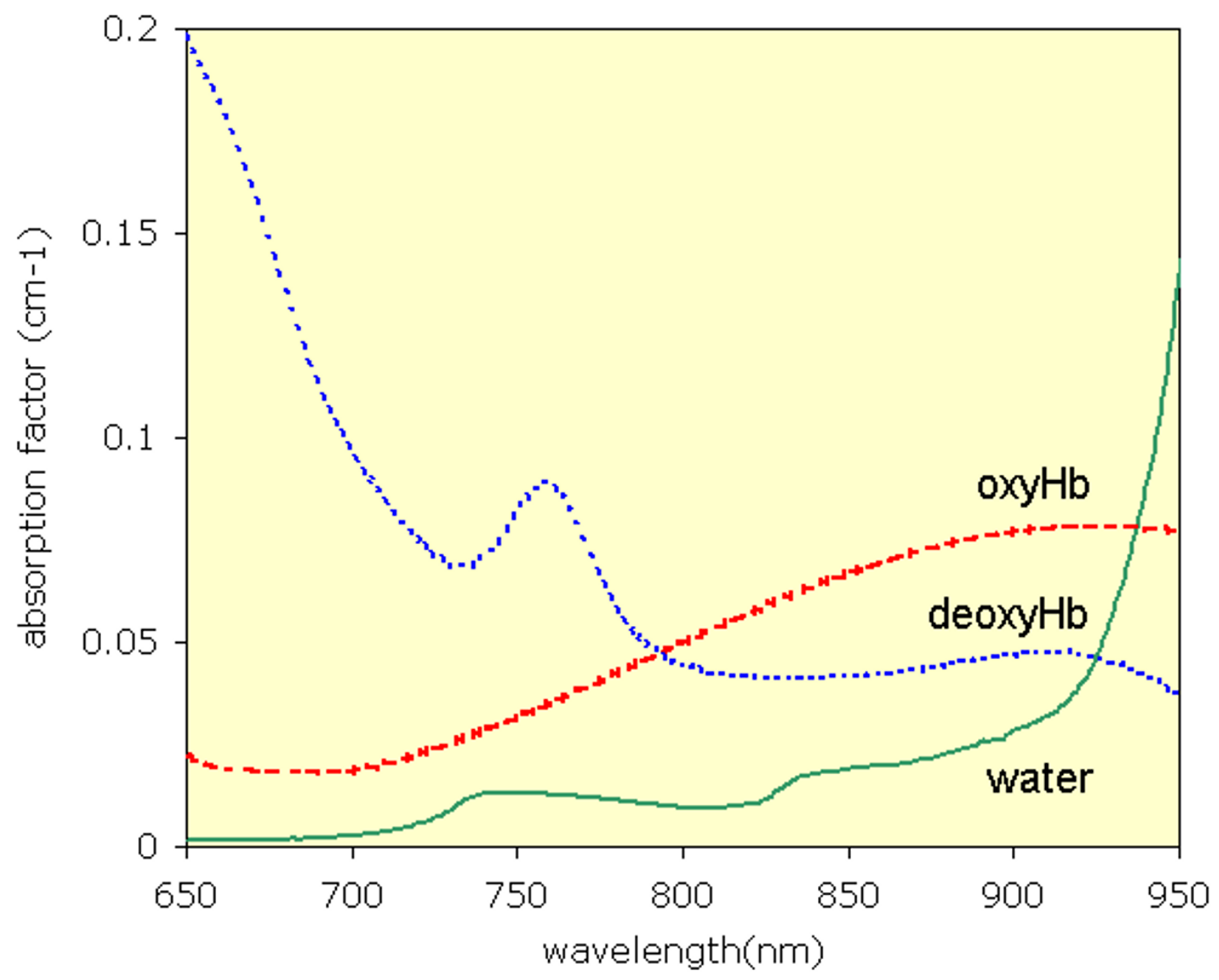

Figure I

The optical window used in diffuse optical measurements

hazards. The effect of NIR light on eye and skin are studied separately since the eye lens focuses the light on the retina. Focused light is stronger in terms of irradiance than nonfocused light. Hence, injury potential increases with focusing. Here, we concentrate on effects of NIR light on skin, since the light source is attached to the skin during diffuse optical measurements.

The main cause of the potential hazard for NIR light shone through the skin is the heating effect. When NIR light is emitted by an LED in direct contact with the skin, there are two sources of heat: radiated NIR light energy absorbed by the skin, and conducted energy, which is caused by the temperature increase in the semiconductor junction inside the LED. Temperature increase due to the radiated energy has been quantified to be less than $0.5^{\circ} \mathrm{C}$ [2]. However, the effect of conducted heat due to the temperature increase in the semiconductor junction has not yet been reported for diffuse optical measurement applications.

Two types of sources are employed to radiate light in NIR range: LEDs and lasers. Standards specific to the risk of the radiated energy for LEDs in direct contact with the skin have not yet been developed. However, standards that govern safety regulations of laser applications can be used for LED source as well [3]. 


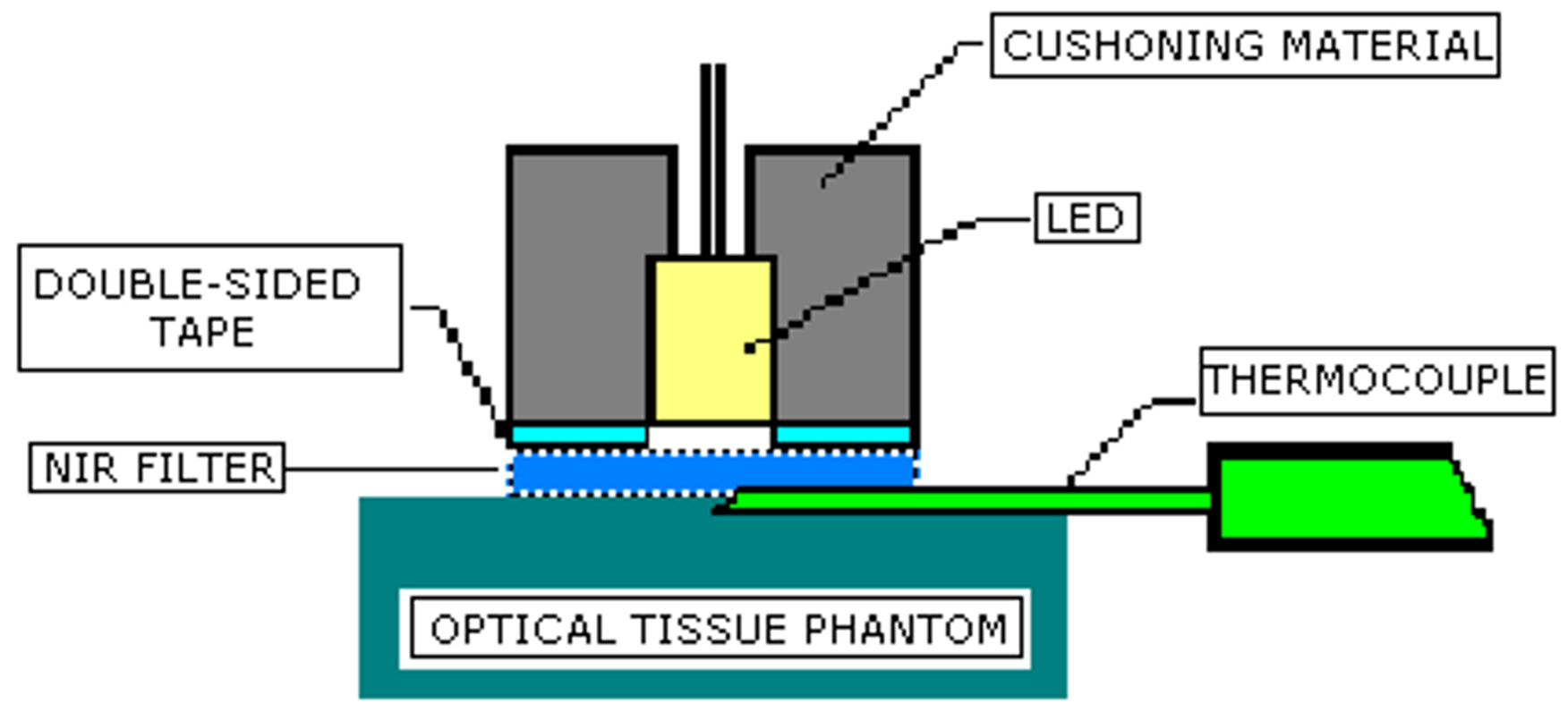

Figure 2

Setup to measure the heating effect of the semiconductor in vitro

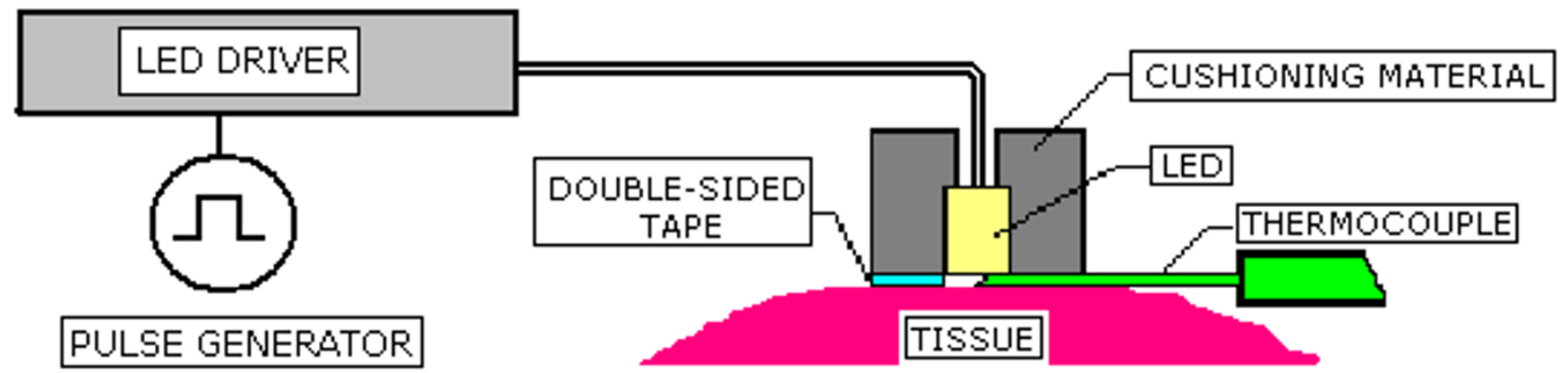

Figure 3

Description of the in-vivo experimental setup

Beyond a certain power level, radiated light energy has the potential to cause serious injuries. This property becomes beneficial in surgical operations for incision and ablation of tissue parts. The thresholds of the damages that can ensue from light energy strongly depend on wavelength, spot size and pulse length of the light [3]. Thus, the effect of each factor should be analyzed to assess the safety of the NIR light.

Light absorption in the tissue varies depending on the wavelength of the light radiated. The most significant effect of the absorbed power is surface heating, which may cause serious burn injuries. This effect is due to absorption of light by water, which has been the key concept behind the tissue ablation and incision using light energy. Especially, wavelengths longer than $950 \mathrm{~nm}$ are strongly absorbed by water. However water is almost transparent to the light in NIR range (fig. 1). In healthy individuals, NIR light is mostly absorbed by blood pigments in deep tissue which does not heat up due to constant circulation. Hence, light in the NIR region does not present the risk of tissue damage that occurs at wavelengths longer than 950 $\mathrm{nm}$ which have been used for laser surgery [3]. NIR wavelengths are not ionizing hence do not carry the risk of 
altering genetic information as may be caused by ultraviolet light.

LEDs and lasers differ in spot size. Spot size defines how well light energy is focused into a single spot. Lasers are inherently collimated. Thus, different spot sizes can be achieved. However, LED light diverges as it leaves the source, so it is difficult to focus all energy into a spot. Different spot sizes may be achieved by means of additional optical apparatus, which is rarely applied in current practice. Hence, spot size is not an issue in LED safety considerations.

Pulse length or in other words light emission duration is another factor determining the potential risk of the radiated light energy. A given amount of energy is turned into more powerful pulses as pulse length decreases. Nanosecond range or shorter pulses are used for ablation in laser surgery operations. The needed short time duration cannot be achieved by LEDs because of the slow response time of the semiconductor junction [4]. Therefore, LEDs do not carry the risk caused by short powerful bursts.

Standards specific to the use of LEDs in direct contact with the skin have not yet been proposed. Though there are some efforts by International Commission on NonIonizing Radiation Protection (ICNIRP), the International Electrotechnical Commission (IEC) and American National Standards Institute (ANSI) to develop regulation about LED hazards, most efforts have been concentrated on eye injury due to radiated energy [5]. LEDs have been regarded safe for eye exposure by a number of studies in the literature $[6,7]$ from a radiated energy perspective. The combined - radiated plus conducted - heating effect of the LEDs on human skin tissue has not been reported yet. In this paper the combined heating effect of the NIR LED in direct contact with skin is analyzed and tested.

\section{Methods}

The measurement setup is illustrated in figure 2 for invitro and figure 3 for in-vivo experiments. An LED (L730805-850-40B32 from Epitex Inc.), that could emit NIR light at three wavelengths of 730, 805, and $850 \mathrm{~nm}$, was employed as a NIR light source. These wavelengths are in the middle of the optical window described in figure 1 . The LED was inserted in a cushioning material that is attached to the surface of the absorbing medium via a medically approved double-sided sticky tape (Adchem Inc.) to ensure good optical contact. An optical phantom with an absorption coefficient of $0.08 \mathrm{~cm}^{-1}$ and reduced scattering coefficient of $12.5 \mathrm{~cm}^{-1}$ is used as the lightabsorbing medium. A thermocouple needle with a 0.1 $\mathrm{mm}$ diameter was inserted between the source and absorbing medium to monitor the temperature change continuously. The needle was connected to the thermo- couple device (Sper Scientific Ltd.). Data was sent to a PC by using RS-232 connection to be stored and plotted continuously. "Testlink 1.1.0.0" software provided by the thermocouple company is used to plot and store the data with sampling frequency of $1 \mathrm{~Hz}$ and temperature resolution of $0.1^{\circ} \mathrm{C}$.

In order to compensate for the effect of the ambient temperature, the ambient temperature was also continuously recorded on the tissue phantom using a second thermocouple $6 \mathrm{~cm}$ away. The change in the baseline due to drifts in ambient temperature was corrected after the experiment.

The heating of the thermocouple needle itself due to NIR absorption was tested in parallel. To do this, NIR light was applied to the needle where the contact to LED was avoided by means of an optical filter which only allows NIR light (NIR-pass filter). It has been observed that the NIR radiation does not significantly heat the thermocouple.

As discussed earlier, the LED source produces two types of heating effects due to conducted and radiated energy. Conducted heat due to the semiconductor junction is measured in-vitro using a NIR-pass filter as shown in figure 2. In this setup, measurements are performed with and without NIR-pass filter. The NIR-pass filter does not heat up due to NIR light absorption since it is transparent to NIR light. When filter is used, the thermocouple measures the temperature increase due to NIR light absorption. When filter is not used, the temperature increase is caused by the combined heating effect of semiconductor junction and NIR light absorption. Therefore, the difference between two readings gives the temperature increase solely due to semiconductor junction heating.

The temperature increase of the semiconductor junction depends on the effective (root mean square) dissipated power of the LED. Different effective powers can be obtained by changing the emitted waveform. DC, pulsating waveform with $12.5 \mathrm{~ms}$ pulse duration and $33.3 \%$ duty cycle and sinusoidal waveform with $1 \mathrm{kHz}$ frequency were tested in-vitro to study the effect of different waveforms. A single wavelength of $730 \mathrm{~nm}$ was used. Peak irradiances between 25 and $50 \mathrm{~mW} / \mathrm{cm}^{2}$ were employed. The crest factor for pulsating waveform and sinusoidal waveform is 1.73 and 1.41 , respectively.

For the pulsating waveform, effective power can be controlled by varying the pulse duration, duty cycle, irradiance and using single or multiple wavelengths. A function generator coupled to the LED driver was used to generate pulsating LED light to study the effect on the temperature of the semiconductor junction. Single wavelength of 730 


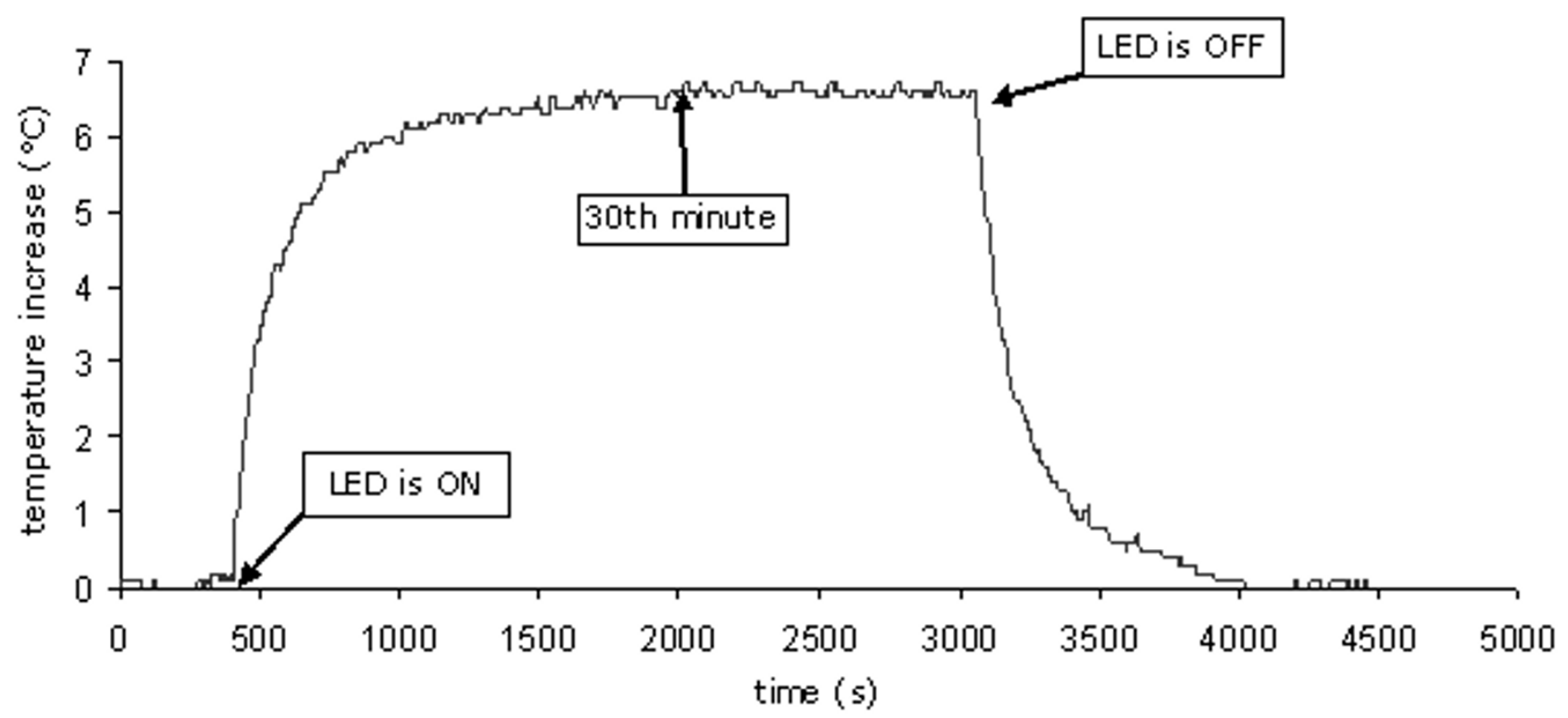

Figure 4

(in vitro) Sample temperature increase result for an experiment

$\mathrm{nm}$ was used. Temperature increase for pulse durations of $12.5,50,100$ and $150 \mathrm{~ms}$, duty cycles of $25 \%, 33.3 \%$, $50 \%$ and $75 \%$ were tested in-vitro. For these duty ratios crest factors are 2, 1.73, 1.41 and 1.15 respectively. The peak value of the pulses was selected to be $37.5 \mathrm{~mW} / \mathrm{cm}^{2}$. This is the power range generally used in diffuse optical measurement applications.

Temperature increase is also affected by the number of different wavelengths used. In continuous wave diffuse optical applications, generally two wavelengths are used to resolve the absorption by two chromophores. Therefore, we tested for the effect of using two wavelengths in-vitro. Wavelengths of 730 and $850 \mathrm{~nm}$ were time multiplexed in a way that only one wavelength was turned on at a time. Pulse duration was $12.5 \mathrm{~ms}$ and duty cycle was $33.3 \%$ which corresponds to a crest factor of 1.73. Peak irradiance was $37.5 \mathrm{~mW} / \mathrm{cm}^{2}$.

In addition to square pulses, $730 \mathrm{~nm}$ and $850 \mathrm{~nm}$ wavelengths with $1 \mathrm{kHz}$ sinusoidal waveform and $90^{\circ}$ phase shift were used in-vitro to study the heating effect of the quadrature phase-modulated system. The peak irradiance of $37.5 \mathrm{~mW} / \mathrm{cm}^{2}$ with a crest factor of 1.73 and wavelengths of 730 and $850 \mathrm{~nm}$ were employed as in the case of square pulses.

The combined effect of the radiated and conductive heat was also tested with an in-vivo set up on three Caucasian adult subjects. All in-vivo studies were carried out under Institutional Review Board (IRB) approval. The LED was directly coupled to the surface of a human arm to observe the in-vivo effect as described in figure 3 . The change in the surface temperature directly beneath the LED was recorded continuously for $12.5 \mathrm{~ms}$ pulses with $37.5 \mathrm{~mW} /$ $\mathrm{cm}^{2}$ irradiance and $33.3 \%$ duty cycle.

During in-vivo experiments, the LED was coupled to the skin using a cushioning material attached to the skin by a medically graded double-sided sticky tape. It is well known that heat increase of the skin is dissipated by sweating mechanism and by heat exchange with air as well as convection via blood flow [8]. The cushioning material that is used to attach the LED to the skin causes pressure and isolation of skin tissue from ambient air. In addition, it blocks sweating by closing the pores and hence contributes to the temperature increase. This was monitored with a secondary thermocouple attached to the skin a few centimeters away from the LED source using the same kind of cushioning material.

In addition to the test setup, the device currently used in Drexel University and University of Pennsylvania with the purpose of functional optical brain imaging using NIR light $[9,10]$ was studied for the heating effect during actual operation with human subjects. The device employs the same kind of LED as used in in-vitro studies. In these experiments, a standard duty cycle of $8.3 \%$, pulse dura- 


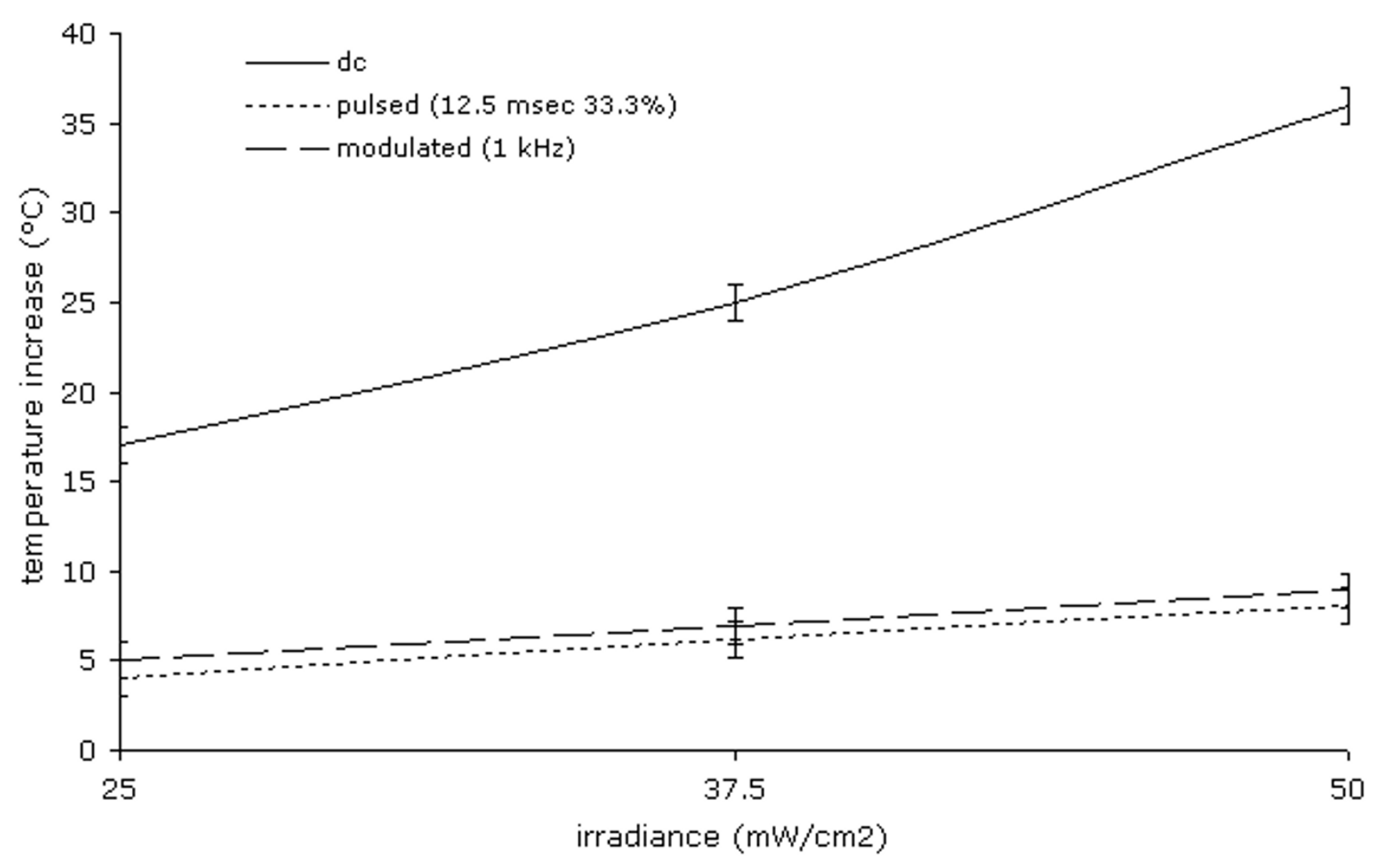

\section{Figure 5}

(in vitro) Temperature increase with varying peak irradiance levels for DC, pulsating and modulated waveforms

tion of $12.5 \mathrm{~ms}$ and irradiance of $37.5 \mathrm{~mW} / \mathrm{cm}^{2}$ were used. The crest factor is 3.47 for such a pulse shape. The temperature rise in the semiconductor junction was tested invitro. The combined heating effect on skin due to both NIR absorption and the heating of semiconductor junction was experimented in-vivo on the human arm and forehead.

In each experiment, data were collected for 35 minutes. The temperature increase after 30 minutes of operation was assumed as the steady state temperature. Data points corresponding to 5 minutes following the 30th minute of the operation were avera ged to determine the resulting temperature increase.

An alternative way to analyze the temperature increase due to absorption of LED emitted NIR light is to compare it with sun emitted NIR light. With the geographic conditions defined by United States Committee on Extension to the Standard Atmosphere, for the 48 contiguous states of the USA over a period of one year as an inclined plane at $37^{\circ}$ tilt toward the equator, facing the sun; i.e., the surface normal points to the sun at an elevation of $48.81^{\circ}$ above the horizon [11], the American Society for Testing and Materials (ASTM) developed and defined a standard terrestrial solar spectral irradiance distribution called ASTM G159 [12]. This standard can be used to calculate the average NIR irradiance emitted by the sun and absorbed by human skin. The average power delivered by the NIR part of the sunlight calculated using this standard is $50 \mathrm{~mW} /$ $\mathrm{cm}^{2}$.

\section{Results}

A typical temperature increase due to the combined effect of conducted and radiated heats of the LED is plotted in figure 4. As seen in this figure, the rise in temperature reached an asymptotical steady state by the 30th minute of operation. This was observed in all of the experiments conducted.

It has been observed that, for a given peak irradiance level, the single wavelength DC waveform increased the temperature of the semiconductor junction more than pulsed and modulated waveforms during in-vitro experiments 


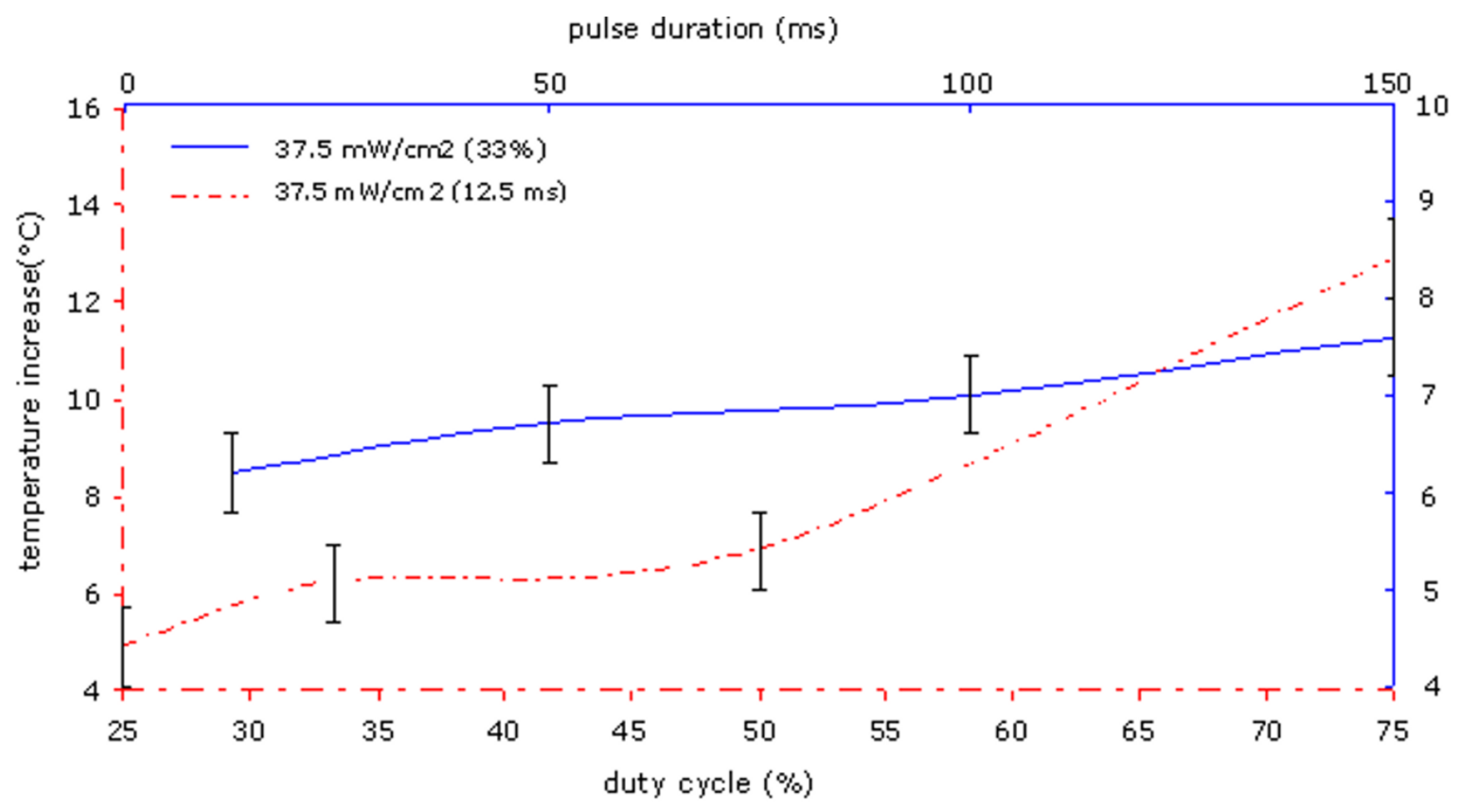

Figure 6

(in vitro) Temperature increase with varying duty cycles and pulse durations

(fig. 5). Pulsed and sinusoidal waveforms caused similar temperature increases. Increase in the peak irradiance of the LED caused higher elevation in the temperature.

The effect of varying pulse characteristics on the temperature of the semiconductor junction can be observed in invitro experiment results. As pulse duration, duty cycle and irradiance were increased the temperature elevation was observed (figure 5,6).

$730 \mathrm{~nm}$ and $850 \mathrm{~nm}$ wavelengths have similar temperature increase effects on the semiconductor junction when they are used one at a time in both sinusoidal and pulsed waveforms. Using both wavelengths simultaneously in a time-switched pulsed manner caused a temperature increase that is approximately equal to the addition of the effect of each wavelength. When two wavelengths with sinusoidal waveform having $90^{\circ}$ phase shift are used simultaneously, temperature increase was approximately the summation of the effect of each wavelength. (fig. 7)

In-vivo and in-vitro results demonstrated a similar trend for increasing irradiance (fig. 8). In-vivo temperature increase results were approximately $1^{\circ} \mathrm{C}$ higher than invitro results. The temperature increase due to the cushion- ing material used to attach the light source was $0.5 \pm$ $0.1^{\circ} \mathrm{C}$, which was caused by blocking skin-air heat exchange and sweating.

Results of in-vitro and in-vivo experiments, where the functional near infrared brain imaging device of Drexel University was used, can be found in figure 8 also. During the in-vivo experiments, temperature rise was observed as in the case of in-vitro results; however the temperature increase was $1-1.5^{\circ} \mathrm{C}$ higher than in the in-vitro results.

\section{Discussion}

Under normal conditions, the main potential hazard of NIR light is caused by tissue heating as a result of light absorption by the skin. When an LED is used as the source of the NIR light in direct contact with the skin, an additional temperature increase occurs due to the heating effect of the semiconductor junction. The combined heating must be considered to assess the safety risk. An understanding of the separate contributions of these two effects will significantly help the design of new generation diffuse optical measurement systems.

The experiments by Ito et al [2], which employed laser light with irradiance levels and wavelength similar to the 


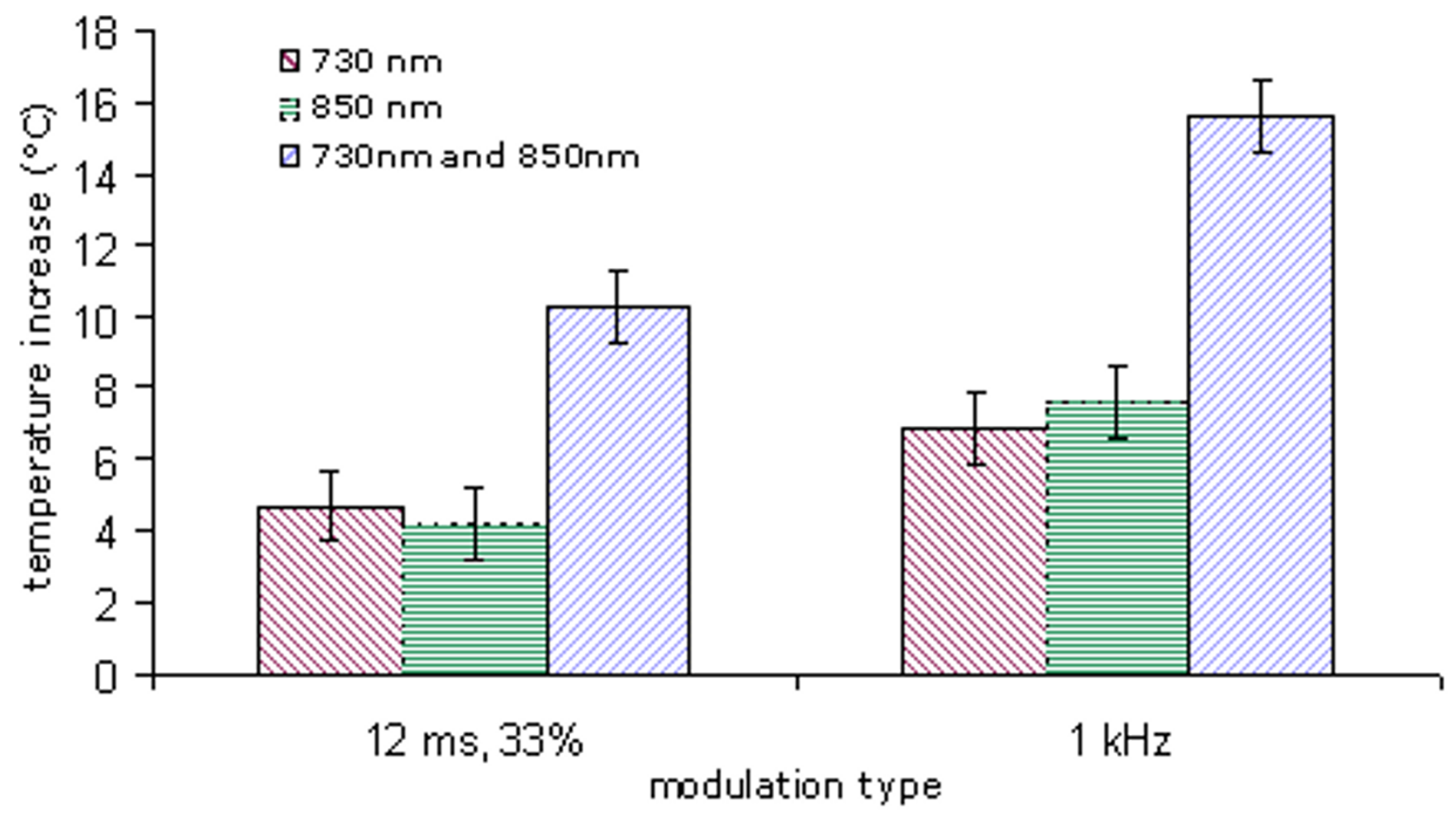

Figure 7

(one wavelength vs. two wavelength) Temperature increase for pulsating and phase modulated waveforms

above experiments, created a temperature increase of 0.3 $\pm 0.2^{\circ} \mathrm{C}$ at the depth of $0.5 \mathrm{~mm}$. This is similar to our findings during in-vitro experiments. In those experiments, they used optical fibers to insulate skin from junction temperature to measure the increase in temperature created only by NIR light absorption.

In in-vitro experiments, we studied the effect of the semiconductor junction. In some cases, the temperature increase due to semiconductor junction can be minimized by using thermal insulators such as double walled glass window. Alternatively, we demonstrated that using sinusoidal or pulsating waveforms results in less temperature rise compared to the case when DC waveform is used for a given amount of power, since the idle duration between two pulses creates a cooling effect. It has also been also observed that the degree of cooling can be controlled by varying the pulse duration, duty cycle, and irradiance, and using single or multiple wavelengths. This is due to the fact that effective (root mean square) dissipated power, which determines the temperature increase, depends on these parameters. As pulse duration, duty cycle and irradiance increase, the effective dissipated power increases causing the temperature to rise.
Further, the same argument applies to heating due to absorption of NIR light by the absorbing medium. During the experiments, although peak irradiance of the pulsating waveforms radiated by LED was between 25 and 50 $\mathrm{mW} / \mathrm{cm}^{2}$, the average irradiance was between 12 and 25 $\mathrm{mW} / \mathrm{cm}^{2}$. This is comparable to the irradiance of the NIR region of the sunlight, which is about $50 \mathrm{~mW} / \mathrm{cm}^{2}$.

It should be noted that temperature increase can be controlled by pulse parameters, while factors such as signal to noise ratio and temporal resolution also depends on these parameters. Therefore, when designing continuous wave diffuse optical measurement systems trade-off between those factors should be considered.

When multiple wavelengths are considered, different wavelengths are emitted by separate semiconductor junctions encapsulated in a single LED package. The semiconductor junction for one wavelength functions independent of others. Thus, the heat generated by each of the LEDs is independent. It follows that, when two wavelengths are employed together, the expected effect is approximately the superposition of temperature increases 


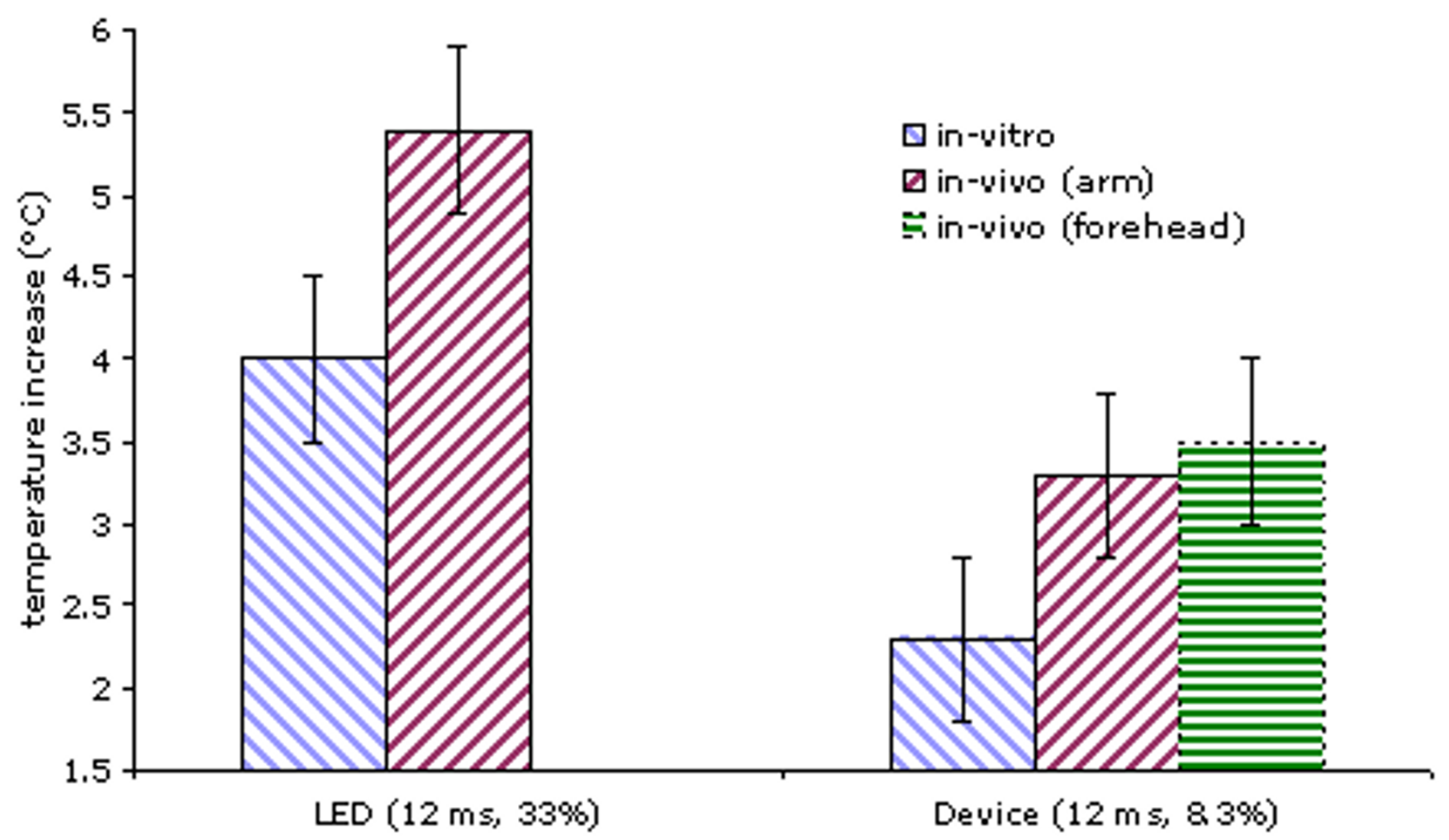

Figure 8

(in vivo vs. in vitro) Temperature increase for set up in fig 3 and for brain-imaging device

observed when they are used separately. This is also supported by experimental results.

Temperature increases due to the semiconductor junction itself varies depending on various LED parameters such as the material used to manufacture the semiconductor junction and the geometry of the package. We used an AlGaAs LED that encapsulates 12 different diodes mounted with AlN heat sink pedestal on TO-5 stem and sealed with a flat glass can. Different temperature increases may be observed for other off-the-shelf LEDs. Therefore, one has to be careful about the quantitative results about the temperature increase due to the semiconductor junction documented in this study. However, qualitative interpretation of the results reported ab ove is valid for all LEDs.

In-vivo experiments demonstrate that the temperature increase on human arm and forehead is $1.5 \pm 0.5^{\circ} \mathrm{C}$ greater than in-vitro results. The first reason for this difference is the temperature increase contributed by absorption of NIR light by tissue that was neglected in in-vitro experiments. In the in-vitro experiments, the only source of the heat was semiconductor junction.
In summary, the observed temperature increase in in-vivo experiments is contributed by heating of the semiconductor junction, heating due to absorption of NIR light and the effect of isolation by the cushioning material. The relative contribution of each heating can be estimated. Based on our experiments and the experiments of Ito et al. [2], temperature increase due to NIR light absorption is less than $0.5^{\circ} \mathrm{C}$. The contribution of the cushioning material, on the other hand, is also less than $0.5^{\circ} \mathrm{C}$. Thus, the temperature increase due to semiconductor junction is in the range of 1 to $10^{\circ} \mathrm{C}$. Therefore, the major source of temperature increase is the semiconductor junction.

When NIR light emitted by LED attached to the skin is shone to the tissue, the main hazard is due to heating, which may cause burn injuries $[13,14]$. It is a well-known fact that the probability of cell death increases when the cell temperatures are sustained above $41^{\circ} \mathrm{C}$ [8]. This value is also the limit value for pulse oximetry applications [15]. Therefore, temperature should be kept under this temperature in order to avoid any burn injury.

Finally, body temperature varies according to the location of the measurement. For instance, the average skin tem- 
perature on the surface of the arm is $32^{\circ} \mathrm{C}$ whereas it is around $35^{\circ} \mathrm{C}$ on the forehead. Thus, the maximum temperature increase can be $9^{\circ} \mathrm{C}$ on the arm whereas it is $6^{\circ} \mathrm{C}$ in the forehead not to exceed the limit of $41^{\circ} \mathrm{C}$. According to this, the maximum allowable temperature increase depends on the location of the application.

\section{Conclusions}

Standards and regulations are required for every medical technology to protect humans from harmful effects. As of this publication, there are no specific standards that ensure the safety of NIR LEDs used in diffuse optical measurement applications, especially when it is placed in direct contact with the skin. Although it is reasonable to use standard limits for laser sources for optical radiation hazards to the skin in the absence of standards specific to LEDs, there is still a need to establish standards for the conducted heat due to the LED. This is particularly important since improper usage of LEDs may cause burn injuries especially in long term monitoring of vulnerable populations such as newborns. In this study, we have assessed the combined effect of conducted and radiated heat in skin caused by NIR emitting LEDs during diffuse optical measurements. The main source of heating is found to be the temperature increase in the semiconductor junction. Elevations up to $10^{\circ} \mathrm{C}$ have been observed. The temperature contribution of NIR absorption by the skin is as low as $0.5^{\circ} \mathrm{C}$ since applied light power is comparable with the power of the NIR region of the sunlight.

When designing diffuse optical systems with LEDs in direct contact with skin, the temperature increase due to semiconductor junction can be minimized by using insulating layers. Alternatively, temperature increase can be controlled by adjusting irradiance, duty cycle and duration of NIR light pulses and frequency of the sinusoids. As discussed in the body of the paper, it is possible to reduce the overall temperature increase down to $1^{\circ} \mathrm{C}$ by shining light in the form of pulses or sinusoids instead of DC waveform.

\section{Authors' contributions}

$\mathrm{AB}$ carried out the in-vivo and in-vitro experiments and drafted the manuscript. BO conceived of the study, and participated in its design and coordination. All authors read and approved the final manuscript.

\section{Acknowledgements}

We acknowledge, with thanks, Dr. Sandy Weininger of Food and Drug Administration (FDA) for helpful comments on the manuscript. This work has been sponsored in part by funds from the Defense Advanced Research Projects Agency (DARPA) Augmented Cognition Program and the Office of Naval Research (ONR), under agreement numbers N000 I 4-02-I-0524 and N000I4-0I-I-0986.

\section{References}

I. Villringer A, Chance B: Non-invasive optical spectroscopy and imaging of human brain function. Trends in Neurosciences 1997, 20(I0):435-42.

2. Ito $\mathrm{Y}$, Kennan RP, Watanabe E, Koizumi H: Assessment of heating effects in skin during continuous wave near infrared spectroscopy. Journal of Biomedical Optics 2000, 5(4):383-90.

3. Sliney DH, Wolbarsht M: Safety With Lasers and Other Optical Sources: A Comprehensive Handbook. New York: Plenum Pub Corp; 1980.

4. Bhattacharya P: Semiconductor Optoelectronic Devices. Englewood Cliffs NJ: Prentice Hall 1996.

5. Sliney DH: ICNIRP Statement on Light Emitting Diodes (LEDs) and Laser Diodes - Implications for Hazard Assessment. Health Physics 2000, 78:744-752.

6. Horak W: Risk Assesment of Light Emitting Diodes. Journal of Laser Applications 1999, I I:2 I-6.

7. Sliney DH: Laser and LED eye hazards: Safety standards. Optics \& Photonic News 1997, 8:31-37.

8. Guyton AC: Textbook of Medical Physiology. Philadelphia: Saunders 2000.

9. Izzetoglu K, Bozkurt A, Yurtsever G, Bunce S: Functional Brain Monitoring via NIR Based Optical Spectroscopy. In Proc. IEEE 29th Annual Northeast Bioengineering Conference: 22-23 March 2003 Newark IEEE; 2003:335-336.

10. Lin Y, Lech G, Nioka S, Intes X, Chance B: Noninvasive, low-noise, fast imaging of blood volume and deoxygenation changes in muscles using light-emitting diode continuous-wave imager. Review of Scientific Instruments 2002, 73(8):3065-3074.

II. United States Committee on Extension to the Standard Atmosphere. U.S. Standard Atmosphere, 1962: ICAO extension to 32 kilometers. Committee on Extension to the Standard Atmosphere Washington DC: US Government Printing Office; 1962.

12. ASTM: Standard Tables for Reference Solar Spectral Irradiance at Air Mass 1.5: Direct Normal and Hemispherical for a $37^{\circ}$ Tilted Surface, Standard GI59-98 West Conshohocken, PA: American Society for Testing and Materials; 1999.

13. Murphy KG, Secunda JA, Rockoff MA: Severe burns from a pulse oximeter. Anesthesiology 1990, 73(2):350-2.

14. Sobel DB: Burning of a neonate due to a pulse oximeter: arterial saturation monitoring. Pediatrics 1992, 89(I): I54-5.

15. Weininger S: Designing a pulse oximeter safety standard. Anesth Analg 2002, 94( I Suppl):S4-7.
Publish with Biomed Central and every scientist can read your work free of charge

"BioMed Central will be the most significant development for disseminating the results of biomedical research in our lifetime. " Sir Paul Nurse, Cancer Research UK

Your research papers will be:

- available free of charge to the entire biomedical community

- peer reviewed and published immediately upon acceptance

- cited in PubMed and archived on PubMed Central

- yours - you keep the copyright

Submit your manuscript here:

http://www.biomedcentral.com/info/publishing_adv.asp
BiolMedcentral 